\title{
Anti-TB Evaluation of Novel 2,3-Dihydroquinazolin- 4(1H)-Ones and in Silico Studies of The Active Compounds
}

\section{Apurba Dutta}

Dibrugarh University

Priyanka Trivedi

CSIR-Central Drug Research Institute: Central Drug Research Institute

Dipshikha Gogoi

Dibrugarh University

Pankaj Chetia

Dibrugarh University

Vinita Chaturvedi

CSIR-Central Drug Research Institute: Central Drug Research Institute

Diganta Sarma ( $\sim$ dsarma22@gmail.com )

Dibrugarh university https://orcid.org/0000-0001-5174-418X

\section{Research Article}

Keywords: Tuberculosis, Anti-tubercular activity, 2,3-Dihydroquinazolin-4(1H)-ones, Molecular docking

Posted Date: March 24th, 2021

DOI: https://doi.org/10.21203/rs.3.rs-341353/v1

License: (9) (i) This work is licensed under a Creative Commons Attribution 4.0 International License.

Read Full License 


\title{
Anti-TB evaluation of novel 2,3-dihydroquinazolin-4(1H)-ones and in silico studies of
}

\section{the active compounds}

Apurba Dutta ${ }^{\mathrm{a}}$, Priyanka Trivedi ${ }^{\mathrm{b}}$, Dipshikha Gogoi ${ }^{\mathrm{c}}$, Pankaj Chetia ${ }^{\mathrm{c}}$, Vinita Chaturvedi ${ }^{\mathrm{b} *}$, Diganta Sarma ${ }^{\text {a* }}$

a Department of Chemistry, Dibrugarh University, Dibrugarh 786004, Assam, India ${ }^{b}$ Biochemistry Division, Central Drug Research Institute, CSIR, Lucknow 226001, India

${ }^{c}$ Department of Life Sciences, Dibrugarh University, Dibrugarh 786004, Assam, India

\begin{abstract}
In vitro anti-tubercular activity of a series of 15 novel 2,3-dihydroquinazolin4(1H)-one analogues were evaluated against Mycobacterium tuberculosis $\mathrm{H}_{37} \mathrm{Ra}$ (ATCC 25177 strain). Among the series, seven compounds showed moderate to good anti-TB activity with minimum inhibitory concentration (MIC) values ranging from 25.0-12.5 $\mu \mathrm{g} / \mathrm{mL}$. Further, in silico experiments were carried out to identify the probable ligand-protein interaction. Molecular docking of the target compounds into the active site of enzymes 1DQY Antigen 85C from Mycobacterium Tuberculosis and 2NSD Enoyl Acyl Carrier Protein Reductase reveals notable information on the possible binding interactions.
\end{abstract}

Keywords: Tuberculosis; Anti-tubercular activity; 2,3-Dihydroquinazolin-4(1H)-ones; Molecular docking

\section{Introduction}

Tuberculosis (TB) a communicable disease, spreads through air droplets containing M. tuberculosis bacilli. TB is treated with a combination of $1^{\text {st }}$ line anti-TB agents, viz. Isoniazid, Rifampicin, Ethambutol and Pyrazinamide. The drug susceptible disease is cured after a 6 months long treatment under DOTs (Directly observed treatment) Program recommended by WHO. The present regimens of TB treatment have many drawbacks such as long treatment duration with drugs that are reported to cause organ toxicity and noncompliance to treatment [1,2]. This may result in the development of drug resistance. To improve the current regimens of $\mathrm{TB}$, medicinal chemists are trying to design and synthesize new drug molecules that may overcome these shortcomings.

Quinazoline is one of the vital N-containing heterocycles bearing a benzene ring and a pyrimidine ring in its structure with chemical formula $\mathrm{C}_{8} \mathrm{H}_{6} \mathrm{~N}_{2}$ [3]. Quinazoline and its analogues appear in more than 100 biologically significant naturally occurring alkaloids, most commonly in the form of quinazolin-4(3H)-one moiety [4]. Medicinal chemists synthesized a variety of quinazoline compounds with different biological activities by introducing various active groups to the quinazoline moiety using developed as well as new synthetic protocols. The potential applications of the quinazoline derivatives in the fields of biology, pesticides and medicine have also been explored. One of the important quinazoline derivatives, deoxyvasicinone (2,3-dihydropyrrolo[2,1-b]quinazolin-9(1H)-one) is an alkaloid 
isolated from the aerial parts of Justicia adhatoda Linn. (Sanskrit- Vasaka), an evergreen sub-herbaceous bush, used extensively as local medicine for cough, cold, bronchitis and asthma [5]. Deoxyvasicinone possesses wide spectrum of biological properties like antimicrobial, anti-inflammatory and antidepressant activities [6-9] as well as very important key intermediate for the synthesis of various natural products such as vasicinone [10], isaindigotone [11] and luotonin A [12]. Because of their diverse biological and pharmacological activities and extensive applications in pharmaceutical, research interest on the synthesis of quinazoline and its derivatives has never faded.

2,3-dihydroquinazolin-4(1H)-one is one of the derivatives of quinazoline that possesses broad range of biological, medicinal and pharmacological activities such as anti-tumor, antibiotic, anti-tubercular, anti-defibrillatory, anti-pyretic, analgesic, anti-hypertonic, diuretic, anti-histamine, anti-depressant, vasodilating agents and many more [13-22]. Since 2,3dihydroquinazolin-4(1H)-one acquire different biological applications, in the present work, we made an attempt to synthesize some novel 2,3-dihydroquinazolin-4(1H)-ones with an aim to get probable novel anti-TB agent(s).

\section{Results and Discussion}

\section{Chemistry}

Our aim was to synthesize a series of novel derivatives of 2,3-dihydroquinazolin-4(1H)-ones and evaluation of their anti-TB potency. Using our method developed recently [23], we have synthesized ten derivatives of novel 2,3-dihydroquinazolin-4(1H)-ones. Afterwards, the work was extended for the construction of some novel bis-2,3-dihydroquinazolin-4(1H)-one analogues via Glaser coupling reaction using copper acetate in which 2a-2e were used as starting precursors [24]. All the five reactions gave the desired novel bis-2,3-dihydroquinazolin-4(1H)-ones in good to excellent yields which was reported in our recent work [23]. From ${ }^{1} \mathrm{H}$ and ${ }^{13} \mathrm{C}$ NMR spectroscopic analysis, it was confirmed that all these novel bis-2,3-dihydroquinazolin-4(1H)-one analogues were $\mathrm{C}_{2}$-symmetric. Literature survey reveals that $\mathrm{C}_{2}$-symmetric molecules have been employed in a number of catalytic reactions as ligands as they limit the different types of side reactions. Therefore, these five $\mathrm{C}_{2}$-symmetric analogues of 2,3-dihydroquinazolin-4(1H)-one are also expected to have extensive application in various organic transformations in coming days. All the synthesized 15 novel 2,3-dihydroquinazolin4(1H)-one compounds are depicted in Scheme 1. 
Scheme 1 Novel analogues of 2,3-dihydroquinazolin-4(1H)-ones

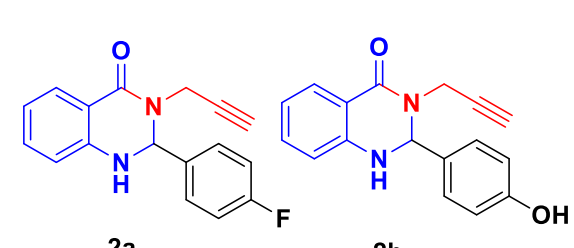<smiles>C#CCN1C(=O)c2ccccc2NC1c1cccc(F)c1</smiles><smiles>C#CCN1C(=O)c2ccccc2NC1c1ccccc1F</smiles><smiles>C=CN1C(=O)c2ccccc2NC1c1c(F)cccc1Cl</smiles><smiles>CSc1ccc(N2C(=O)c3ccccc3NC2c2ccccc2C)cc1N1C(=O)c2ccccc2NC1c1ccccc1N1C(=O)c2ccccc2NC1c1ccccc1F</smiles><smiles>O=C1c2ccccc2NC(c2ccc(F)cc2)N1CC#CC#CCN1C(=O)c2ccccc2NC1c1ccc(F)cc1</smiles><smiles>O=C1c2ccccc2NC(c2ccc(O)cc2)N1CC#CC#CCN1C(=O)c2ccccc2NC1c1ccc(O)cc1</smiles><smiles>C#CCN1C(=O)c2ccccc2NC1c1cccc(F)c1</smiles>

$3 c$

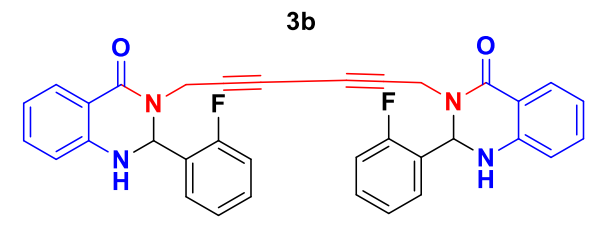

$3 d$

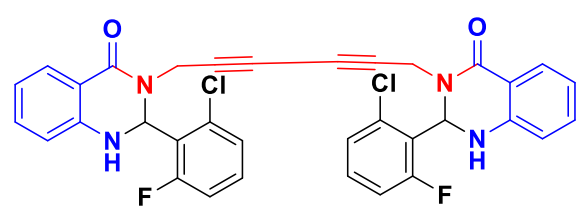

$3 e$

\section{Biological evaluation}

Proportion (agar dilution) assay [25] was used for anti-TB activity determination of the synthesized compounds in terms of Minimum Inhibitory Concentrations (MICs) against MTB $\mathrm{H}_{37} \mathrm{Ra}$ (ATCC 25177). Different concentrations of the compounds ranging from 25.0 to $3.125 \mu \mathrm{g} / \mathrm{mL}$ were tested to determine the MICs. From this experiment one compound ( $2 \mathrm{~b})$ was found to display appreciable anti-TB activity with MIC $12.5 \mu \mathrm{g} / \mathrm{mL}$. Other six compounds (2e, $\mathbf{2 h}, \mathbf{2} \mathbf{j}, \mathbf{3 b}, \mathbf{3 d}$ and $\mathbf{3 e}$ ) showed activity at the concentration of $25.0 \mu \mathrm{g} / \mathrm{mL}$. The remaining 8 compounds did not show activity up to $25.0 \mu \mathrm{g} / \mathrm{mL}$, the highest concentration tested. In the present work, Ethambutol, an anti-TB drug, was used as a positive control. 
Table 1 Anti-tubercular activity of newly synthesized compounds

\begin{tabular}{|c|c|c|c|c|}
\hline Compounds & Structure & $C \log P^{a}$ & $\mathrm{CMR}^{\mathrm{a}}$ & $\operatorname{MIC}(\mu \mathrm{g} / \mathrm{mL})$ \\
\hline $2 a$ & & 3.6068 & 7.9272 & $>25$ \\
\hline $2 \mathrm{~b}$ & & 2.7968 & 8.0678 & 12.5 \\
\hline $2 c$ & & 2.7968 & 8.0678 & $>25$ \\
\hline 2d & & 2.7968 & 8.0678 & $>25$ \\
\hline $2 e$ & & 4.3198 & 8.4186 & 25 \\
\hline $2 f$ & & 5.4208 & 10.5199 & $>25$ \\
\hline $2 \mathrm{~g}$ & & 5.4208 & 10.5199 & $>25$ \\
\hline $2 \mathrm{~h}$ & & 3.9338 & 11.0775 & 25 \\
\hline
\end{tabular}


$2 \mathbf{i}$

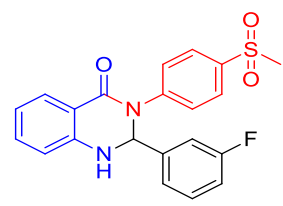

2j

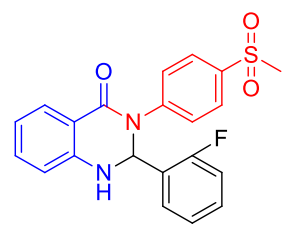

3.2208

10.5861

25

$3 \mathbf{a}$

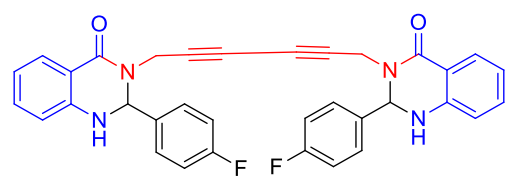

5.6196

15.829

$>25$

$3 b$<smiles>O=C1c2ccccc2NC(c2ccc(O)cc2)N1CC#CC#CCN1C(=O)c2ccccc2NC1c1ccc(O)cc1</smiles>

3.9996

16.1042

25

$3 c$<smiles>O=C1c2ccccc2NC(c2cccc(F)c2)N1CC#CC#CCN1C(=O)c2ccccc2NC1c1cccc(F)c1</smiles>

5.6196

15.829

$>25$

3d

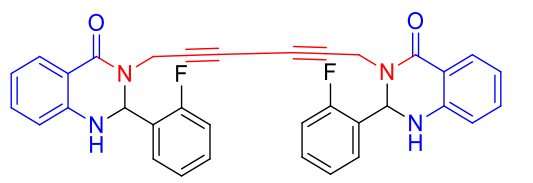

5.6196

15.829

25

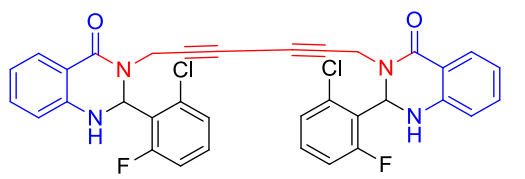

7.0456

16.8116

25

Ethambutol

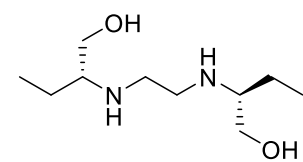

$0.1188 \quad 5.859$

2.0

${ }^{\mathrm{a} C}$ Log P and CMR were calculated using Chemdraw Ultra 12.0 software by Cambridge Soft.

\section{Molecular docking study}

In silico experiments were carried out to identify the ligand-protein interaction. In this work, LibDock was used to evaluate the binding affinities between the active compounds and the enzymes 2NSD Enoyl Acyl Carrier Protein Reductase and 1DQY Antigen 85C from $M$. 
tuberculosis. Among all the compounds tested against the Enoyl Acyl Carrier Protein Reductase, five compounds exhibited good docking scores (Table 2). Among the five compounds, compound 3b showed the lowest CDOCKER interaction energy score of $54.5525 \mathrm{kcal} / \mathrm{mol}$. Apart from the binding energy, it has formed one conventional H-bond with the residue Phe41. Conventional H-bonds are the major contributors for the stability in binding of protein and compounds. Apart from H-bond, it also formed some other interaction such as $\mathrm{C}-\mathrm{H}$ bond, unfavorable donor-donor, pi-donor H-bond, pi-sigma, pi-pi T-shaped and pi-alkyl. The 2D and 3D structures of the docked complex is depicted in Figure 1.

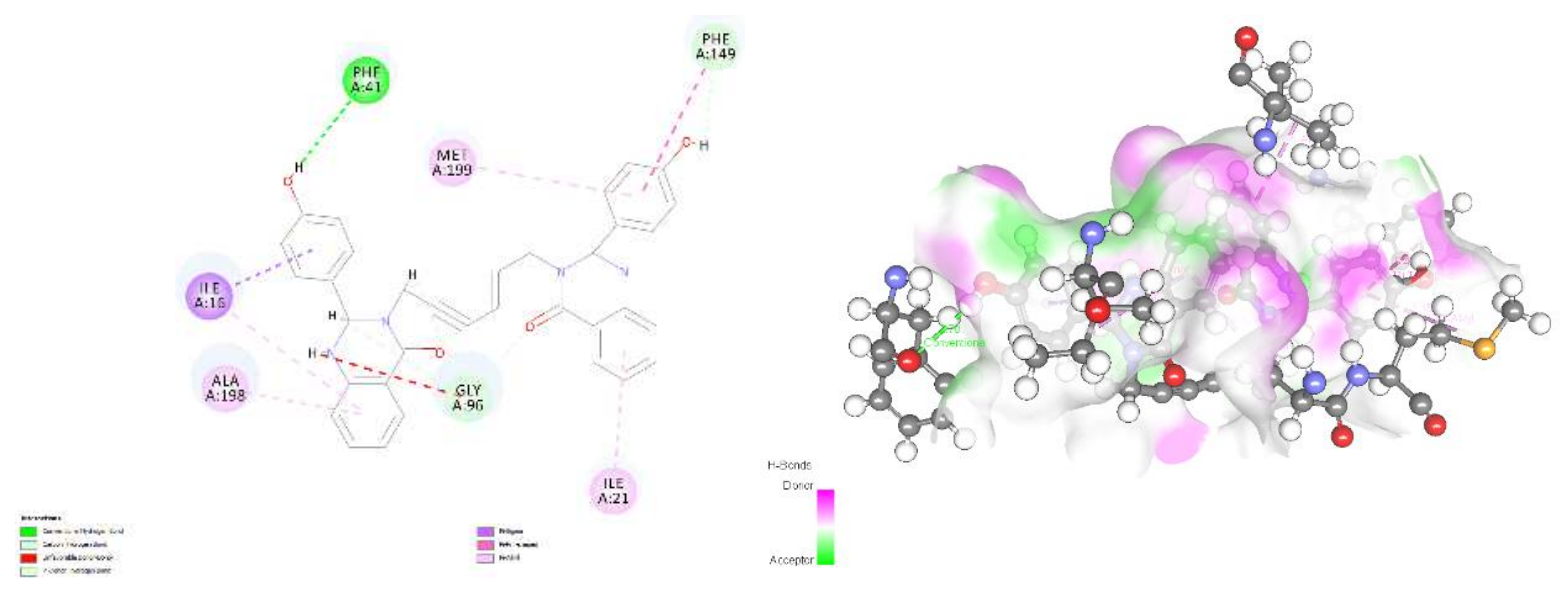

Figure $12 \mathrm{D}$ and 3D structure of $\mathbf{3 b}$ with target 2NSD

Table 2 Docking score of 2NSD with five compounds and potential amino acids of the protein interacting with ligands along with the interaction

\begin{tabular}{|c|c|c|c|c|}
\hline Comp. & $\begin{array}{c}\text { CDOCKER } \\
\text { energy } \\
\text { (Kcal } / \text { mol })\end{array}$ & $\begin{array}{c}\text { CDOCKER } \\
\text { interaction energy } \\
(\text { Kcal } / \mathbf{m o l})\end{array}$ & Interacting amino acid & Interaction \\
\hline $\begin{array}{c}\mathbf{2 b} \\
(\mathrm{POSE} 2)\end{array}$ & -30.8509 & -36.2778 & $\begin{array}{l}\text { Lys165, MET147, Ala191, } \\
\text { Gly14, Ser20, Ile21, Ala198 }\end{array}$ & $\begin{array}{l}\text { Conventional H-bond, } \\
\text { C-H bond, pi-alkyl }\end{array}$ \\
\hline $\begin{array}{c}\mathbf{2 e} \\
(\mathrm{POSE} 2)\end{array}$ & -23.921 & -34.346 & $\begin{array}{l}\text { Ile21, Met161, Thr196, } \\
\text { Ala198, Met147 }\end{array}$ & $\begin{array}{l}\text { Conventional H-bond, } \\
\text { pi-alkyl }\end{array}$ \\
\hline $\begin{array}{c}\mathbf{2 j} \\
(\mathrm{POSE} 2)\end{array}$ & -27.1222 & -40.1077 & $\begin{array}{l}\text { Ile16, Ile21, Ser94, Lys165, } \\
\text { Met161, Ala198 }\end{array}$ & $\begin{array}{l}\text { Conventional H-bond, } \\
\text { C-H bond, pi-sulfur, } \\
\text { pi-alkyl }\end{array}$ \\
\hline $\begin{array}{c}\mathbf{2 h} \\
(\mathrm{POSE} 2)\end{array}$ & -22.9169 & -39.1576 & $\begin{array}{l}\text { Phe149, Met199, Ala198, } \\
\text { Ile21 }\end{array}$ & $\begin{array}{l}\text { C-H bond, pi-pi } \mathrm{T}- \\
\text { shaped, pi-alkyl }\end{array}$ \\
\hline
\end{tabular}




\begin{tabular}{ccclc}
\hline 3b & -40.574 & -54.5525 & Phe41, Met199, Phe149, & Conventional H-bond, \\
(POSE2) & & Ile21, Gly96, Ala198, Ile16 & C-H & bond, \\
& & unfavorable donor- \\
& & donor, pi-donor H- \\
& & bond, pi-sigma, pi-pi \\
& & T-shaped, pi-alkyl \\
\hline
\end{tabular}

Similarly an in silico experiment was executed for these seven compounds on 1DQY Antigen 85C from $M$. tuberculosis for exploring the anti-TB potency of the synthesized novel analogues. Only two compounds tested against the Antigen 85C showed good docking scores (Table 3). Compound 2b showed the lowest CDOCKER interaction energy score of -37.0648 $\mathrm{kcal} / \mathrm{mol}$. Apart from the binding energy it has formed 2 conventional $\mathrm{H}$-bonds with the residues Asp38 and Arg41 which are lying within the active pocket of the protein. Apart from $\mathrm{H}$-bonds, it also formed some other interaction such as $\mathrm{C}-\mathrm{H}$ bond, pi-pi T-shaped and pialkyl. The 3D and 2D structures of the docked complex is shown in Figure 2.

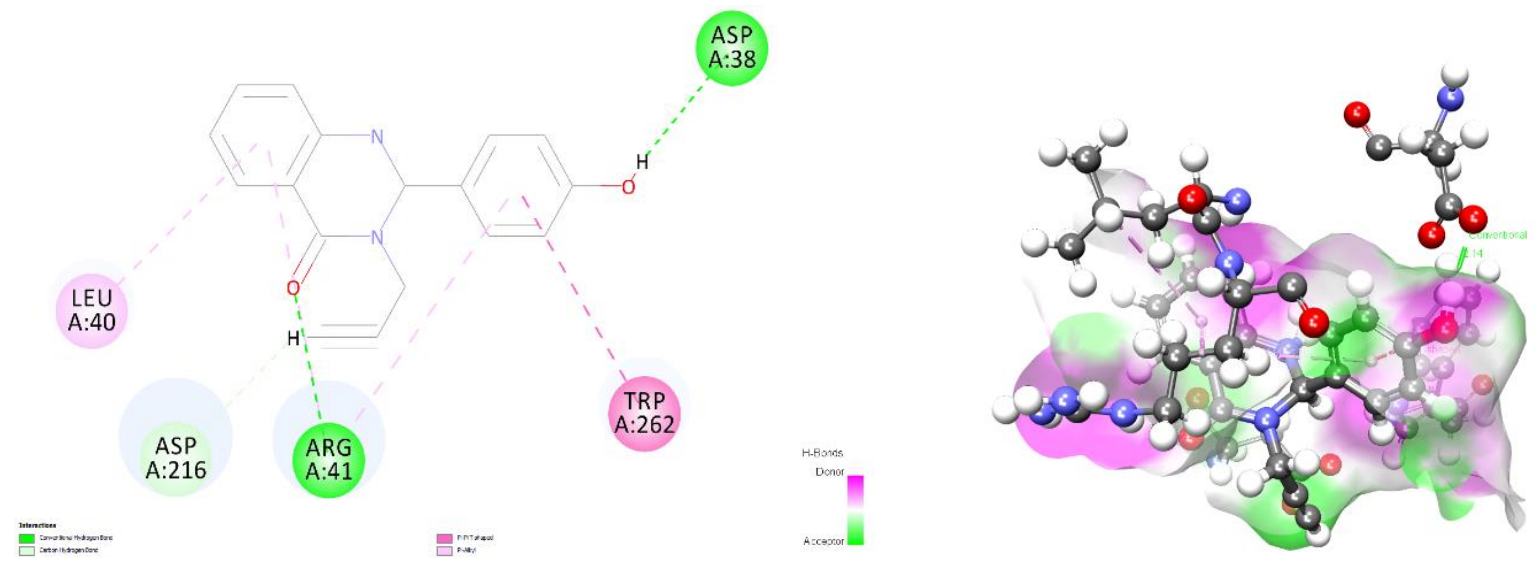

Figure 2 2D and 3D structures of $\mathbf{2 b}$ with target 1DQY 
Table 2 Docking score of 1DQY with two compounds and potential amino acids of the protein interacting with ligands along with the interaction

\begin{tabular}{cccll}
\hline Comp. & $\begin{array}{c}\text { CDOCKER } \\
\text { energy } \\
(\mathbf{K c a l} / \mathbf{m o l})\end{array}$ & $\begin{array}{c}\text { CDOCKER } \\
\text { interaction energy } \\
(\mathbf{K c a l} / \mathbf{m o l})\end{array}$ & $\begin{array}{c}\text { Interacting amino } \\
\text { acid }\end{array}$ & Interaction \\
\hline $\mathbf{2 b}$ & -30.7248 & -37.0648 & Asp38, Trp262, Arg41, & Conventional \\
$($ POSE2) & & Asp216, Leu40 & H-bond, C-H \\
& & & & bond, pi-pi T \\
& & & Lys165, & Mhaped, pi-alkyl \\
2e & -16.9365 & -31.0986 & Ala191, Gly14, Ser20, & H-bond, C-H \\
$($ POSE2) & & & Ile21, Ala198 & bond, pi-alkyl \\
\hline
\end{tabular}

\section{Conclusion}

In summary, we designed and successfully executed synthesis of some novel derivatives of 2,3-dihydroquinazolin-4(1H)-one including five $\mathrm{C}_{2}$ symmetric bis-2,3dihydroquinazolin-4(1H)-ones. The newly synthesized compounds were evaluated for their potent in vitro anti-tubercular activity. Among the 15 novel compounds, seven were identified as potent anti-TB agents with MIC in the range of $25.0-12.5 \mu \mathrm{g} / \mathrm{mL}$. The most potent compound, $\mathbf{2} \mathbf{b}$ inhibited the growth of $M T B$ at the concentration of $12.5 \mu \mathrm{g} / \mathrm{mL}$. Further, molecular docking of the target compounds into the active site of enzymes 1DQY Antigen 85C from Mycobacterium Tuberculosis and 2NSD Enoyl Acyl Carrier Protein Reductase reveals notable information on the possible binding interactions. These findings suggest that the newly designed compounds may be considered as potential agents for antiTB drug discovery.

\section{Experimental}

\section{General method for synthesis of 2,3-dihydroquinazolin-4(1H)-ones (2a-2j) [23]}

Isatoic anhydride $(1 \mathrm{mmol})$, amines $(1.2 \mathrm{mmol})$, carbonyls $(1.2 \mathrm{mmol})$ and $\left[\left\{\mathrm{SiO}_{2}-\right.\right.$ (acac) $\}_{3} \mathrm{Fe}^{\mathrm{III}} \mathrm{Cl}_{3}(20 \mathrm{mg}, 32.24 \mathrm{ppm} \mathrm{Fe})$ in water $(2 \mathrm{~mL})$ were stirred in a flask equipped with a condenser at $80{ }^{\circ} \mathrm{C}$. On completion (monitored by TLC under UV light), the resulting mixture was cooled to room temperature and then the mixture was extracted with ethyl acetate $(3 \times 10 \mathrm{~mL})$ and washed with $\mathrm{H}_{2} \mathrm{O}(3 \times 10 \mathrm{~mL})$. The combined extract was dried over anhydrous $\mathrm{Na}_{2} \mathrm{SO}_{4}$. The solvent of filtrate was removed with the aid of a rotary evaporator. The product was purified by column chromatography on silica gel using petroleum 
ether/ethyl acetate $(3: 1 \mathrm{v} / \mathrm{v})$ as eluent to obtain the pure novel compounds. The products were then characterized by ESI-MS, ${ }^{1} \mathrm{H}$ NMR and ${ }^{13} \mathrm{C}$ NMR analyses.

\section{General procedure for the synthesis of compounds (3a-3e) [23]}

A mixture of 2a-2e $(1 \mathrm{mmol})$, piperidine $(1 \mathrm{mmol})$ and $\mathrm{Cu}(\mathrm{OAc})_{2} \cdot \mathrm{H}_{2} \mathrm{O}(10 \mathrm{~mol} \%)$ in $\mathrm{CH}_{2} \mathrm{Cl}_{2}(2 \mathrm{~mL})$ was stirred in an open air at room temperature for 10 hours. On completion (monitored by TLC under UV light), the resulting mixture was extracted with ethyl acetate (3 $\mathrm{x} 10 \mathrm{~mL})$ and washed with water $(3 \mathrm{x} 10 \mathrm{~mL})$. The combined extract was dried over anhydrous $\mathrm{Na}_{2} \mathrm{SO}_{4}$. The solvent of filtrate was removed with the aid of a rotary evaporator. The crude product was purified via column chromatography on silica gel using petroleum ether/ethyl acetate $(3: 1 \mathrm{v} / \mathrm{v})$ as eluent to obtain the pure novel compounds. The compounds were then characterized by ESI-MS, ${ }^{1} \mathrm{H}$ NMR and ${ }^{13} \mathrm{C}$ NMR analyses.

\section{2-(4-Fluorophenyl)-3-(prop-2-yn-1-yl)-2,3-dihydroquinazolin-4(1H)-one (2a)}

Brown solid; 98\% yield; Mp: 136-138 ${ }^{\circ} \mathrm{C}$; IR $(\mathrm{KBr}) \mathrm{cm}^{-1}$ : 3302, 2927, 2365, 2346, 1634, 1506, 1427, 1304, 1226, 1154, 860, 842, 750, 683, 651, 622, 546, 525; ${ }^{1} \mathrm{H}$ NMR (400 MHz, DMSO- $\left.d_{6}\right) \delta 7.67(\mathrm{dd}, J=7.7,1.4 \mathrm{~Hz}, 1 \mathrm{H}), 7.42$ (ddd, $\left.J=13.0,8.2,2.0 \mathrm{~Hz}, 3 \mathrm{H}\right), 7.23$ (dddd, $J=19.2,8.9,7.0,1.8 \mathrm{~Hz}, 2 \mathrm{H}), 6.73-6.66(\mathrm{~m}, 2 \mathrm{H}), 5.98$ (s, 1H), 3.64 (s, 2H), 3.16 (t, $J=2.4$ $\mathrm{Hz}, 1 \mathrm{H}) ;{ }^{13} \mathrm{C}$ NMR $(100 \mathrm{MHz}, \mathrm{DMSO}-d 6) \delta 163.39,162.02,146.38,136.16,133.72,128.71$, $128.63,127.60,117.37,115.44,115.23,114.34,113.92,78.93,74.45,69.63,33.06$; MS (ESI) calcd for $\mathrm{C}_{17} \mathrm{H}_{13} \mathrm{FN}_{2} \mathrm{O}[\mathrm{M}+\mathrm{H}]^{+}$281.1090; found 281.1100.

\section{2-(4-Hydroxyphenyl)-3-(prop-2-yn-1-yl)-2,3-dihydroquinazolin-4(1H)-one (2b)}

Brown solid; 95\% yield; Mp: 58-60 ${ }^{\circ} \mathrm{C}$; IR $(\mathrm{KBr}) \mathrm{cm}^{-1}: 3358,3253,2926,2854,1634,1611$, 1500, 1456, 1428, 1405, 1266, 1232, 1167, 986, 840, 686, 593, 554, 526; ${ }^{1}$ H NMR (400 MHz, DMSO-d6) $\delta 7.66(\mathrm{~d}, J=7.9 \mathrm{~Hz}, 1 \mathrm{H}), 7.22(\mathrm{dd}, J=13.2,7.9 \mathrm{~Hz}, 4 \mathrm{H}), 6.83-6.57(\mathrm{~m}$, $3 \mathrm{H}), 5.84(\mathrm{~s}, 1 \mathrm{H}), 4.64(\mathrm{~d}, J=17.3 \mathrm{~Hz}, 2 \mathrm{H}), 3.11(\mathrm{~s}, 1 \mathrm{H}) ;{ }^{13} \mathrm{C}$ NMR (100 MHz, DMSO-d6) $\delta$ $162.91,158.38,147.32,134.16,130.30,128.58,128.14,117.69,115.71,114.78,114.34$, 79.59, 74.63, 70.78, 33.10 ; MS (ESI) calcd for $\mathrm{C}_{17} \mathrm{H}_{14} \mathrm{~N}_{2} \mathrm{O}_{2}[\mathrm{M}+\mathrm{H}]^{+}$279.1133; found 279.1139.

\section{2-(3-Fluorophenyl)-3-(prop-2-yn-1-yl)-2,3-dihydroquinazolin-4(1H)-one (2c)}

White solid; $98 \%$ yield; Mp: 80-82 ${ }^{\circ} \mathrm{C}$; IR (KBr) cm${ }^{-1}: 3429,3295,1640,1620,1589,1528$, 1488, 1452, 1428, 1360, 1307, 1259, 1158, 1134, 1035, 919, 860, 797, 748, 656, 631, 585, $521 ;{ }^{1} \mathrm{H}$ NMR (400 MHz, DMSO-d6) $\delta 7.66(\mathrm{~d}, J=1.4 \mathrm{~Hz}, 1 \mathrm{H}), 7.49-7.12(\mathrm{~m}, 4 \mathrm{H}), 6.71-6.69$ $(\mathrm{m}, 1 \mathrm{H}), 6.50-6.44(\mathrm{~m}, 2 \mathrm{H}), 6.01(\mathrm{~s}, 1 \mathrm{H}), 4.08(\mathrm{~s}, 2 \mathrm{H}), 3.06(\mathrm{t}, J=2.5 \mathrm{~Hz}, 1 \mathrm{H}) ;{ }^{13} \mathrm{C} \mathrm{NMR}$ $\left(100 \mathrm{MHz}, \mathrm{DMSO}-d_{6}\right) \delta 168.51,149.81,146.23,142.96,133.76,131.90,128.01,127.58$, 
122.38, 117.51, 116.39, 114.49, 113.67, 81.59, 74.59, 72.41, 33.41 ; MS (ESI) calcd for $\mathrm{C}_{17} \mathrm{H}_{13} \mathrm{FN}_{2} \mathrm{O}[\mathrm{M}+\mathrm{H}]^{+}$281.1090; found 281.1085.

\section{2-(2-Fluorophenyl)-3-(prop-2-yn-1-yl)-2,3-dihydroquinazolin-4(1H)-one (2d)}

Light brown solid; $95 \%$ yield; Mp: $116-118{ }^{\circ} \mathrm{C}$; IR (KBr) $\mathrm{cm}^{-1}: 3326,3247,2927,2850$, 2123, 1632, 1513, 1488, 1426, 1401, 1339, 1307, 1275, 1235, 1158, 1033, 988, 946, 843, 759, 693, 620, 550, 521; ${ }^{1} \mathrm{H}$ NMR (400 MHz, DMSO-d6) $\delta 7.70(\mathrm{dd}, J=7.7,1.5 \mathrm{~Hz}, 1 \mathrm{H})$, 7.41-7.12 (m, 6H), 6.74-6.66 (m, 2H), $6.30(\mathrm{~s}, 1 \mathrm{H}), 3.72(\mathrm{~s}, 2 \mathrm{H}), 3.15(\mathrm{t}, J=2.4 \mathrm{~Hz}, 1 \mathrm{H}) ;{ }^{13} \mathrm{C}$ NMR (100 MHz, DMSO-d6) $\delta$ 161.94, 160.80, 146.04, 133.72, 130.80, 127.53, 124.48, 124.45, 117.36, 116.01, 115.80, 114.35, 113.64, 78.61, 74.59, 64.59, 32.95 ; MS (ESI) calcd for $\mathrm{C}_{17} \mathrm{H}_{13} \mathrm{FN}_{2} \mathrm{O}[\mathrm{M}+\mathrm{H}]^{+}$281.1090; found 281.1085.

\section{2-(2-Chloro-6-fluorophenyl)-3-(prop-2-yn-1-yl)-2,3-dihydroquinazolin-4(1H)-one (2e)}

Pale yellow solid; $92 \%$ yield; Mp: $72-74{ }^{\circ} \mathrm{C}$; IR (KBr) cm ${ }^{-1}: 3432,2956,2923,2854,2331$, 1637, 1466, 1380, 1164, 1077, 1020, 985, 916, 799; ${ }^{1} \mathrm{H}$ NMR (400 MHz, DMSO-d6) $\delta 8.59$ (s, 1H), $7.66(\mathrm{dd}, J=7.7,1.5 \mathrm{~Hz}, 1 \mathrm{H}), 7.47-7.21(\mathrm{~m}, 3 \mathrm{H}), 6.70-6.41(\mathrm{~m}, 3 \mathrm{H}), 5.78(\mathrm{~s}, 1 \mathrm{H})$, 3.97 (s, 2H), 3.05 (s, $1 \mathrm{H}) ;{ }^{13} \mathrm{C}$ NMR (100 MHz, DMSO-d6) $\delta 163.70,161.83,150.25,134.07$, 132.46, 128.54, 127.82, 126.73, 115.08, 114.21, 113.89, 113.17, 82.12, 78.83, 72.92, 32.74 ; MS (ESI) calcd for $\mathrm{C}_{17} \mathrm{H}_{12} \mathrm{ClFN}_{2} \mathrm{O}[\mathrm{M}+\mathrm{H}]^{+}$315.0700; found 315.0745.

\section{2-(3-Fluorophenyl)-3-(4-(methylthio)phenyl)-2,3-dihydroquinazolin-4(1H)-one (2f)}

Light brown solid; $62 \%$ yield; Mp: $174-176{ }^{\circ} \mathrm{C}$; IR (KBr) $\mathrm{cm}^{-1}: 3422,3296,2923,2852$, $1642,1614,1587,1487,1448,1387,1292,1241,1122,1020,928,871,828,773,753,689$, 669, 543, 518; ${ }^{1} \mathrm{H}$ NMR (500 MHz, DMSO-d6) $\delta$ 7.74-7.68 (m, 2H), 7.30-7.20 (m, 8H), 6.79$6.74(\mathrm{~m}, 2 \mathrm{H}), 6.32$ (s, 1H), 2.45 (s, 3H); ${ }^{13} \mathrm{C}$ NMR (125 MHz, DMSO-d6) $\delta 162.49,161.42$, $146.77,143.97,137.86,136.18,134.25,130.86,128.34,127.09$, 126.46, 123.02, 123.00, $118.13,115.69,115.57,115.52,115.20,113.95,72.25,15.14 ;$ MS (ESI) calcd for $\mathrm{C}_{21} \mathrm{H}_{17} \mathrm{FN}_{2} \mathrm{OS}[\mathrm{M}+\mathrm{H}]^{+}$365.1124; found 365.1119.

\section{2-(2-Fluorophenyl)-3-(4-(methylthio)phenyl)-2,3-dihydroquinazolin-4(1H)-one (2g)}

Light brown solid; $85 \%$ yield; Mp: $158-160{ }^{\circ} \mathrm{C}$; IR (KBr) $\mathrm{cm}^{-1}$ : 3292, 2923, 2367, 2345, 1637, 1610, 1509, 1493, 1401, 1363, 1322, 1260, 1231, 1162, 1089, 1051, 1020, 954, 820, 765, 687, 623, 529, 465, 434; ${ }^{1} \mathrm{H}$ NMR (400 MHz, DMSO-d6) $\delta$ 7.84-7.09 (m, 10H), 6.79$6.71(\mathrm{~m}, 2 \mathrm{H}), 6.51(\mathrm{~s}, 1 \mathrm{H}), 2.43(\mathrm{~s}, 3 \mathrm{H}) ;{ }^{13} \mathrm{C} \mathrm{NMR}$ (100 MHz, DMSO-d6) $\delta 162.21,160.46$, $146.38,137.04,136.30,133.77,130.70,130.62$, 128.14, 128.11, 127.84, 127.38, 125.97, $124.45,124.42,117.55,115.90,115.69,114.59,67.71,14.63$; MS (ESI) calcd for $\mathrm{C}_{21} \mathrm{H}_{17} \mathrm{FN}_{2} \mathrm{OS}[\mathrm{M}+\mathrm{H}]^{+}$365.1124; found 365.1119. 
2-(2-Chloro-6-fluorophenyl)-3-(4-(methylsulfonyl)phenyl)-2,3-dihydroquinazolin-4(1H)one (2h)

Light brown solid; 58\% yield; Mp: 90-92 ${ }^{\circ} \mathrm{C}$; IR (KBr) $\mathrm{cm}^{-1}: 3473,3375,2923,2852,1670$, 1620, 1588, 1568, 1518, 1489, 1453, 1420, 1301, 1247, 1160, 1113, 1050, 910, 860, 782, 749, 718, 657, 530; ${ }^{1} \mathrm{H}$ NMR (400 MHz, DMSO- $\left.d 6\right) \delta 7.84-7.62(\mathrm{~m}, 3 \mathrm{H}), 7.20(\mathrm{t}, J=7.4 \mathrm{~Hz}$, $3 \mathrm{H}), 6.71(\mathrm{~d}, J=8.3 \mathrm{~Hz}, 2 \mathrm{H}), 6.48(\mathrm{t}, J=7.4 \mathrm{~Hz}, 3 \mathrm{H}), 5.68(\mathrm{~s}, 1 \mathrm{H}), 3.49$ (s, 3H); ${ }^{13} \mathrm{C} \mathrm{NMR}$ (100 MHz, DMSO-d6) $\delta$ 169.97, 166.54, 151.89, 145.52, 134.81, 134.20, 131.61, 127.07, 126.12, 124.98, 123.42, 116.75, 115.06, 110.03, 77.87, 48.11; MS (ESI) calcd for $\mathrm{C}_{21} \mathrm{H}_{16} \mathrm{ClFN}_{2} \mathrm{O}_{3} \mathrm{~S}[\mathrm{M}+\mathrm{H}]^{+} 431.0632$; found 431.0645 .

\section{2-(3-Fluorophenyl)-3-(4-(methylsulfonyl)phenyl)-2,3-dihydroquinazolin-4(1H)-one (2i)}

Pale yellow solid; 55\% yield; Mp: $116-118{ }^{\circ} \mathrm{C}$; IR (KBr) $\mathrm{cm}^{-1}: 3473,3375,2923,1673,1620$, 1589, 1563, 1486, 1420, 1302, 1250, 1159, 1117, 1053, 920, 751, 657, 530; ${ }^{1} \mathrm{H}$ NMR (500 MHz, DMSO-d6) $\delta$ 7.83-7.79 (m, 2H), $7.70-7.67(\mathrm{~m}, 2 \mathrm{H}), 7.22(\mathrm{ddd}, J=8.5,7.1,1.6 \mathrm{~Hz}$, $2 \mathrm{H}), 6.74-6.73(\mathrm{~m}, 5 \mathrm{H}), 6.51-6.48(\mathrm{~m}, 1 \mathrm{H}), 5.74(\mathrm{~s}, 1 \mathrm{H}), 3.09(\mathrm{~s}, 3 \mathrm{H}) ;{ }^{13} \mathrm{C} \mathrm{NMR}(125 \mathrm{MHz}$, DMSO- $\left.d_{6}\right) \delta 163.28,161.34,151.85,134.66,134.05,131.49,131.18,131.12,125.78,125.76$, 123.28, 120.30, 116.66, 116.17, 115.99, 114.88, 109.92, 73.03, 45.80 ; MS (ESI) calcd for $\mathrm{C}_{21} \mathrm{H}_{17} \mathrm{FN}_{2} \mathrm{O}_{3} \mathrm{~S}[\mathrm{M}+\mathrm{H}]^{+}$397.1022; found 397.1045.

\section{2-(2-Fluorophenyl)-3-(4-(methylsulfonyl)phenyl)-2,3-dihydroquinazolin-4(1H)-one (2j)}

Pale yellow solid; 56\% yield; Mp: 112-114 ${ }^{\circ} \mathrm{C}$; IR (KBr) cm $\mathrm{cm}^{-1}: 3474,3375,2922,2854,1674$, $1615,1588,1564,1487,1421,1304,1248,1160,1116,1053,920,859,750,708,658,530$; ${ }^{1} \mathrm{H}$ NMR (400 MHz, DMSO-d6) $\delta$ 7.92-7.76 (m, 2H), $7.64(\mathrm{dd}, J=19.1,7.1 \mathrm{~Hz}, 1 \mathrm{H}), 7.24$ $(\mathrm{dt}, J=15.1,7.8 \mathrm{~Hz}, 3 \mathrm{H}), 6.71(\mathrm{~d}, J=8.3 \mathrm{~Hz}, 3 \mathrm{H}), 6.48(\mathrm{t}, J=7.5 \mathrm{~Hz}, 3 \mathrm{H}), 5.83(\mathrm{~s}, 1 \mathrm{H}), 3.55$ (s, 3H); ${ }^{13} \mathrm{C}$ NMR (100 MHz, DMSO-d6) $\delta$ 162.86, 160.30, 151.87, 135.24, 135.15, 134.80, 134.21 , 133.08, 132.36, 131.61, 124.94, 124.91, 123.41, 117.49, 117.27, 116.76, 115.07, 110.01, 76.02, 47.39; $\mathrm{MS}$ (ESI) calcd for $\mathrm{C}_{21} \mathrm{H}_{17} \mathrm{FN}_{2} \mathrm{O}_{3} \mathrm{~S}[\mathrm{M}+\mathrm{H}]^{+}$397.1022; found 397.1056.

\section{3,3'-(Hexa-2,4-diyne-1,6-diyl)bis(2-(4-fluorophenyl)-2,3-dihydroquinazolin-4(1H)-one)}

(3a)

Light brown solid; $70 \%$ yield; Mp: $144-146{ }^{\circ} \mathrm{C}$; IR (KBr) $\mathrm{cm}^{-1}: 3305,2922,1641,1621$, $1509,1418,1395,1333,1297,1226,1157,1014,978,838,784,754,695,660,601,529 ;{ }^{1} \mathrm{H}$ NMR (400 MHz, DMSO-d6) $\delta 7.65(\mathrm{~d}, J=7.5 \mathrm{~Hz}, 2 \mathrm{H}), 7.39(\mathrm{~d}, J=5.7 \mathrm{~Hz}, 2 \mathrm{H}), 7.21(\mathrm{dt}, J=$ 16.9, $7.9 \mathrm{~Hz}, 8 \mathrm{H}), 6.68(\mathrm{dd}, J=13.3,7.2 \mathrm{~Hz}, 4 \mathrm{H}), 5.94(\mathrm{~s}, 2 \mathrm{H}), 4.08(\mathrm{~s}, 4 \mathrm{H}) ;{ }^{13} \mathrm{C} \mathrm{NMR}(100$ MHz, DMSO-d6) $\delta 162.72,161.55,147.02,136.53,134.41,129.31,129.23,128.15,118.01$, 
116.00, 115.79, 114.94, 114.28, 75.06, 70.68, 67.28, 34.46 ; MS (ESI) calcd for $\mathrm{C}_{34} \mathrm{H}_{24} \mathrm{~F}_{2} \mathrm{~N}_{4} \mathrm{O}_{2}[\mathrm{M}+\mathrm{H}]^{+}$559.1945; found 559.1951.

3,3'-(Hexa-2,4-diyne-1,6-diyl)bis(2-(4-hydroxyphenyl)-2,3-dihydroquinazolin-4(1H)-one) (3b)

Brown solid; $80 \%$ yield; Mp: 152-154 ${ }^{\circ} \mathrm{C}$; IR (KBr) cm ${ }^{-1}: 3419,2959,2927,2858,2340$, 1633, 1612, 1512, 1488, 1336, 1235, 1168, 923, 797, 752, 694, 659; ${ }^{1} \mathrm{H}$ NMR (400 MHz, DMSO-d6) $\delta 9.60(\mathrm{~s}, 1 \mathrm{H}), 7.72-6.84(\mathrm{~m}, 12 \mathrm{H}), 6.71(\mathrm{dd}, J=31.0,7.3 \mathrm{~Hz}, 4 \mathrm{H}), 5.80(\mathrm{~s}, 2 \mathrm{H})$, 4.73 (s, 4H); ${ }^{13} \mathrm{C}$ NMR (100 MHz, DMSO- $\left.d 6\right) \delta 160.79,156.26,145.20,132.04,128.18$, $126.46,126.01,115.56,113.59,112.66,112.62,112.22,77.47,72.51,68.65,30.98$; MS (ESI) calcd for $\mathrm{C}_{34} \mathrm{H}_{26} \mathrm{~N}_{4} \mathrm{O}_{4}[\mathrm{M}+\mathrm{H}]^{+}$555.2032; found 555.2039.

\section{3,3'-(Hexa-2,4-diyne-1,6-diyl)bis(2-(3-fluorophenyl)-2,3-dihydroquinazolin-4(1H)-one)}

(3c)

Brown solid; 85\% yield; Mp: 96-98 ${ }^{\circ} \mathrm{C}$; IR (KBr) cm${ }^{-1}: 3331,3064,2927,2853,2340,1633$, $1615,1590,1515,1485,1255,1159,1032,872,752,695,658,522 ;{ }^{1} \mathrm{H}$ NMR $(400 \mathrm{MHz}$, DMSO-d6) $\delta$ 7.66-7.35 (m, 4H), 7.26-7.11 (m, 4H), $6.68(\mathrm{~d}, J=7.9 \mathrm{~Hz}, 6 \mathrm{H}), 6.47$ (dd, $J=$ 19.7, $12.4 \mathrm{~Hz}, 2 \mathrm{H}), 5.95$ (s, 2H), 4.08 (s, 4H); ${ }^{13} \mathrm{C}$ NMR (100 MHz, DMSO-d6) $\delta$ 162.57, $161.24,150.36,134.43,132.61,131.17,128.54,128.12,122.89,118.13,116.98,115.12$, 113.91, 76.98, 70.64, 66.13, 29.30 ; MS (ESI) calcd for $\mathrm{C}_{34} \mathrm{H}_{24} \mathrm{~F}_{2} \mathrm{~N}_{4} \mathrm{O}_{2}[\mathrm{M}+\mathrm{H}]^{+}$559.1945; found 559.1951.

\section{3,3'-(Hexa-2,4-diyne-1,6-diyl)bis(2-(2-fluorophenyl)-2,3-dihydroquinazolin-4(1H)-one)} (3d)

Brown solid; 90\% yield; Mp: 86-88 ${ }^{\circ} \mathrm{C}$; IR (KBr) cm ${ }^{-1}: 3300,2926,2856,1642,1614,1488$, 1449, 1420, 1335, 1156, 1097, 1028, 928, 753, 695, 661, 523; ${ }^{1} \mathrm{H}$ NMR (400 MHz, DMSOd6) $\delta$ 7.73-7.57 (m, 6H), 7.47-7.07 (m, 6H), $6.68(\mathrm{~d}, J=7.3 \mathrm{~Hz}, 4 \mathrm{H}), 6.22(\mathrm{~s}, 2 \mathrm{H}), 3.98(\mathrm{~s}$, $4 \mathrm{H}) ;{ }^{13} \mathrm{C}$ NMR (100 MHz, DMSO- $\left.d_{6}\right) \delta 162.61,160.77,146.69,135.66,134.39,131.43$, $131.35,128.44,128.24,128.07,127.91,126.87,117.96,116.55,116.33,114.92,114.03$, 79.61, 74.78, 65.82, 34.49 ; MS (ESI) calcd for $\mathrm{C}_{34} \mathrm{H}_{24} \mathrm{~F}_{2} \mathrm{~N}_{4} \mathrm{O}_{2}[\mathrm{M}+\mathrm{H}]^{+}$559.1945; found 559.1951 .

\section{3,3'-(Hexa-2,4-diyne-1,6-diyl)bis(2-(2-chloro-6-fluorophenyl)-2,3-dihydroquinazolin- 4(1H)-one) (3e)}

Brown solid; 50\% yield; Mp: 66-68 ${ }^{\circ} \mathrm{C}$; IR (KBr) cm ${ }^{-1}: 3421,2959,2923,2853,2332,1636$, 1541, 1467, 1381, 1162, 1077, 985, 918, 803, 749; ${ }^{1} \mathrm{H}$ NMR (400 MHz, DMSO-d6) $\delta 8.65$ (s, 2H), $7.45(\mathrm{~d}, J=7.7 \mathrm{~Hz}, 2 \mathrm{H}), 7.13(\mathrm{t}, J=7.6 \mathrm{~Hz}, 6 \mathrm{H}), 6.69(\mathrm{~d}, J=8.2 \mathrm{~Hz}, 2 \mathrm{H}), 6.47(\mathrm{dd}, J=$ 18.6, $11.1 \mathrm{~Hz}, 4 \mathrm{H}), 5.84,(\mathrm{~s}, 2 \mathrm{H}), 4.09$ (s, 4H); ${ }^{13} \mathrm{C}$ NMR (100 MHz, DMSO-d6) $\delta 169.16$, 
$169.08,150.31,132.57,128.55,127.79,116.94,115.10,113.92,76.97,66.17,60.22,29.32$;

MS (ESI) calcd for $\mathrm{C}_{34} \mathrm{H}_{22} \mathrm{Cl}_{2} \mathrm{~F}_{2} \mathrm{~N}_{4} \mathrm{O}_{2}[\mathrm{M}+\mathrm{H}]^{+}$627.1166; found 627.1172.

\section{Evaluation of in vitro anti-tubercular activity of compounds}

Initially all the synthesized compounds were screened against M. tuberculosis $\mathrm{H}_{37} \mathrm{Ra}$ (ATCC 25177 strain) at the single concentration of $25 \mu \mathrm{g} / \mathrm{mL}$. The active compounds from the above screening were further tested to determine MIC using Agar Proportion assay. To make stocks $(5 \mathrm{mg} / \mathrm{mL})$, the compounds were dissolved in DMSO. Serial two fold dilutions were also made in DMSO from the stocks. $0.1 \mathrm{~mL}$ of compound or DMSO (negative control) or anti-TB drugs (positive controls) was added to $1.9 \mathrm{~mL} \mathrm{MB7H10} \mathrm{agar} \mathrm{medium} \mathrm{(in} \mathrm{tubes,}$ temperature $45-50{ }^{\circ} \mathrm{C}$, with OADC supplement, final concentration $10 \%$ ). Then the contents were mixed and allowed to solidify as slants. Three week old culture of M. tuberculosis $\mathrm{H}_{37} \mathrm{Ra}$ was harvested from L-J medium and its suspension $(1 \mathrm{mg} / \mathrm{mL}$, equivalent to approximately $10^{8}$ bacilli) was prepared in normal saline containing $0.05 \%$ Tween-80. $10 \mu \mathrm{l}$ of 1:10 dilution of this suspension $\left(\sim 10^{5}\right.$ bacilli) was inoculated into each tube for 4 weeks at the temperature of $37^{\circ} \mathrm{C}$. The lowest concentration of a compound up to which there was no visible growth of bacilli was its Minimal Inhibitory Concentration (MIC). Ethambutol was used as reference drug.

\section{CDocker algorithim in Discovery studio}

The Dock Ligands (CDOCKER) protocol is an implementation of the CDOCKER algorithim. It allows running a refinement docking of any number of ligands with a single protein receptor. CDOCKER is a grid based molecular docking method that employs CHARMm. The receptor is held rigid while the ligands are allowed to flex during the refinement. For predocked ligands, prior knowledge of binding site is not required. It is possible, however, to specify the ligand placement in the active site using a binding site sphere. Random ligand conformations are generated from the initial ligand structure through high temperature molecular dynamics, followed by random rotations. The random conformations are refined by grid based (GRID 1) simulated annealing and a final grid based or full force field minimization. CDOCKER uses a detailed atomic force field that is comprised of accuracy of automated MD docking with a soft-core potential over Monte Carlo (MC) Simulations and Genetic Alogorithim (GA) in searching a large configuration space. 


\section{Force-field and grid}

The grid origin is situated at the centre of the active sites of the protein. A +1 point charge probe is used to map electrostatic interactions to the grid. The grid's vdW interactions are generated in one of two ways. The energies and forces are generated for the soft-core potentials in the heating-cooling stages and with normal non bond potentials in the final minimization step. The soft-core is generally approximated by the below function:

$$
E_{i j}\left(r_{i j}\right)=E_{\max }-a * r^{b_{i j}} \text { if }\left|E^{*}{ }_{i j}\right|>\frac{\mid E m a x}{2}
$$

where $\mathrm{E}^{*_{\mathrm{ij}}}$ is the energy of regular non bond ( $\mathrm{vdW}$ or electrostatic) potential. The coefficients $a$ and $b$ were extracted from two equations that express equality of regular and soft potential and forces at the switching distance.

\section{Acknowledgments}

DS is thankful to Department of Biotechnology, Ministry of Science and Technology, New Delhi, India for a research grant (BT/PR24684/NER/95/810/2017). AD thanks DBT, New Delhi for Research Fellowship. The financial assistance of UGC-SAP programme to the Department of Chemistry, Dibrugarh University is also gratefully acknowledged.

\section{Conflict of interest}

The authors declare no competing interests.

\section{References}

1. Sotgiu, G.; Centis, R.; D'ambrosio, L.; Migliori, G. B. Cold Spring Harb. Perspect. Med., 2015, 5, a017822.

2. Singh, P.; Mishra, A. K.; Malonia, S. K.; Chauhan, D. S.; Sharma, V. D.; Venkatesan, K.; Katoch, V. M. J. Commun. Dis., 2006, 38, 288.

3. Srivastava, S.; Srivastava, S. Int. J. Pharm. Sci., 2015, 6, 1206.

4. Mhaske, S. B.; Argade, N. P. Tetrahedron, 2006, 62, 9787.

5. Mehta, D. R.; Naravane, J. S.; Desai, R. M. J. Org. Chem., 1963, 28, 445.

6. Amin, A. H.; Mehta, D. R. Nature, 1959, 183, 1317.

7. Joshi, B. S.; Newton, M. G.; Lee, D. W.; Barber, A. D.; Pelletier, S. W. Tetrahedron Asymmetry, 1996, 7, 25.

8. Atal, C. K. In Chemistry and Pharmacology of Vasicine. Raj Bandu Industrial Co, Delhi, 1980.

9. Ghosal, S.; Chauhan, P. S. R. B.; Mehta, R. Phytochemistry, 1975, 14, 830. 
10. Al-Shamma, A.; Drake, S.; Flynn, D. L.; Mitscher, L. A.; Park, Y. H.; Rao, G. S. R.; Simpson, A.; Swayze, J. K.; Veysoglu, T.; Wu, S. S. J. Nat. Prod., 1981, 44, 745.

11. Kamal, A.; Ramana, K. V.; Rao, M. V. J. Org. Chem., 2001, 66, 997.

12. Molina, P.; Tarraga, A.; Gonzalez-Tejero, A. Synthesis, 2000, 11, 1523.

13. Zhang, H.; Liu, H.; Luo, X.; Wang, Y.; Liu, Y.; Jin, H.; Liu, Z.; Yang, W.; Yu, P;, Zhang, L.; Zhang, L. Eur. J. Med. Chem., 2018, 152, 235.

14. Nagaladinne, N.; Hindustan, A.; Nayakanti, D. Indian J. Pharm. Sci., 2020, 82, 984.

15. Marvania, B.; Lee, P. C.; Chaniyara, R.; Dong, H.; Suman, S.; Kakadiya, R.; Su, T. L. Bioorg. Med. Chem., 2011, 19, 1987.

16. El-Azab, A. S.; ElTahir, K. E. Bioorg. Med. Chem. Lett., 2012, 22, 1879.

17. Maurya, H. K.; Verma, R.; Alam, S.; Pandey, S.; Pathak, V.; Sharma, S.; Gupta, A. Bioorg. Med. Chem. Lett., 2013, 23, 5844.

18. Dukat, M.; Alix, K.; Worsham, J.; Khatri, S.; Schulte, M. K. Bioorg. Med. Chem. Lett., 2013, 23, 5945.

19. Akester, J. N.; Njaria, P.; Nchinda, A.; Le Manach, C.; Myrick, A.; Singh, V.; Lawrence, N.; Njoroge, M.; Taylor, D.; Moosa, A.; Smith, A. J. ACS Infect. Dis., 2020, 6, 1951.

20. Jafari, E.; Khajouei, M. R.; Hassanzadeh, F.; Hakimelahi, G. H.; Khodarahmi, G. A. Res. Pharm. Sci., 2016, 11, 1.

21. Couturier, C.; Lai, C.; Pellet, A.; Upton, A.; Kaneko, T.; Perron, C.; Cogo, E.; Menegotto, J.; Bauer, A.; Scheiper, B.; Lagrange, S.; Bacque, E. Bioorg. Med. Chem. Lett., 2016, 26, 5290.

22. Maddali, N. K.; Viswanath, I. K.; Murthy, Y. L. N.; Bera, R.; Takhi, M.; Rao, N. S.; Gudla, V. Med. Chem. Res., 2019, 28, 559-570.

23. Dutta, A.; Trivedi, P.; Kulashetra,A.; Kumar, A.; Chaturvedi, V.; Sarma, D. Applied Organometallic Chem., 2021, 35, e6116.

24. Balaraman, K.; Kesavan, V. Synthesis, 2010, 20, 3461.

25. McClachy, J. K. Lab Med., 1978, 9, 47. 
Figures

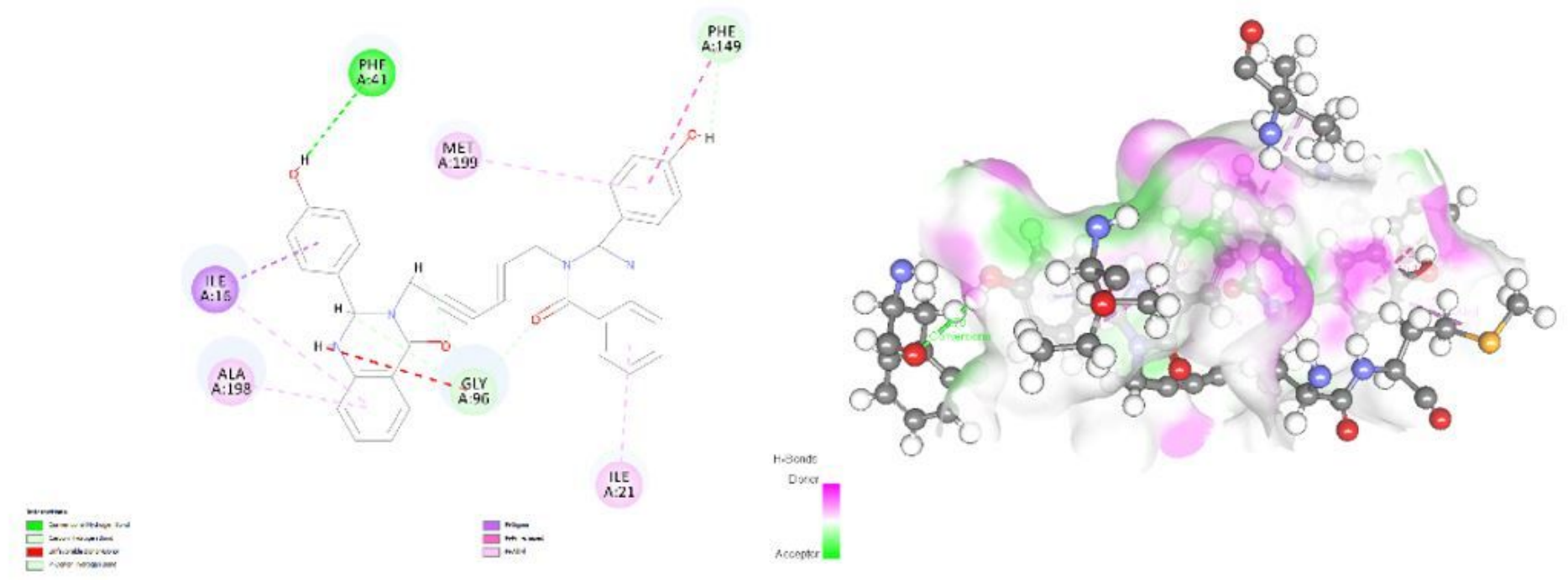

Figure 1

$2 \mathrm{D}$ and $3 \mathrm{D}$ structure of $3 \mathrm{~b}$ with target $2 \mathrm{NSD}$ 
<smiles>C#CCN1C(=O)c2ccccc2NC1c1c(F)cccc1C1Nc2ccccc2C(=O)N1CC#CCN1C(=O)c2ccccc2NC1c1cccc(F)c1</smiles><smiles>CSc1ccc(N2C(=O)c3ccccc3NC(c3cccc(F)c3)N2c2ccc(S(C)(=O)=O)cc2)cc1</smiles><smiles>O=C1c2ccccc2NC(c2ccc(F)cc2)N1CC#CC#CCN1C(=O)c2ccccc2NC1c1ccc(F)cc1</smiles><smiles>O=C1c2ccccc2NC(c2ccc(O)cc2)N1CC#CC#CCN1C(=O)c2ccccc2NC1c1ccc(O)cc1</smiles><smiles>O=C1c2ccccc2NC(c2cccc(F)c2)N1CC#CC#CCN1C(=O)c2ccccc2NC1c1cccc(F)c1</smiles><smiles>O=C1c2ccccc2NC(c2ccccc2F)N1CC#CC#CCN1C(=O)c2ccccc2NC1c1ccccc1F</smiles>

$3 c$<smiles>O=C1c2ccccc2NC(c2c(F)cccc2Cl)N1CC#CC#CCN1C(=O)c2ccccc2NC1c1c(F)cccc1Cl</smiles>

Figure 2

Novel analogues of 2,3-dihydroquinazolin-4(1H)-ones 

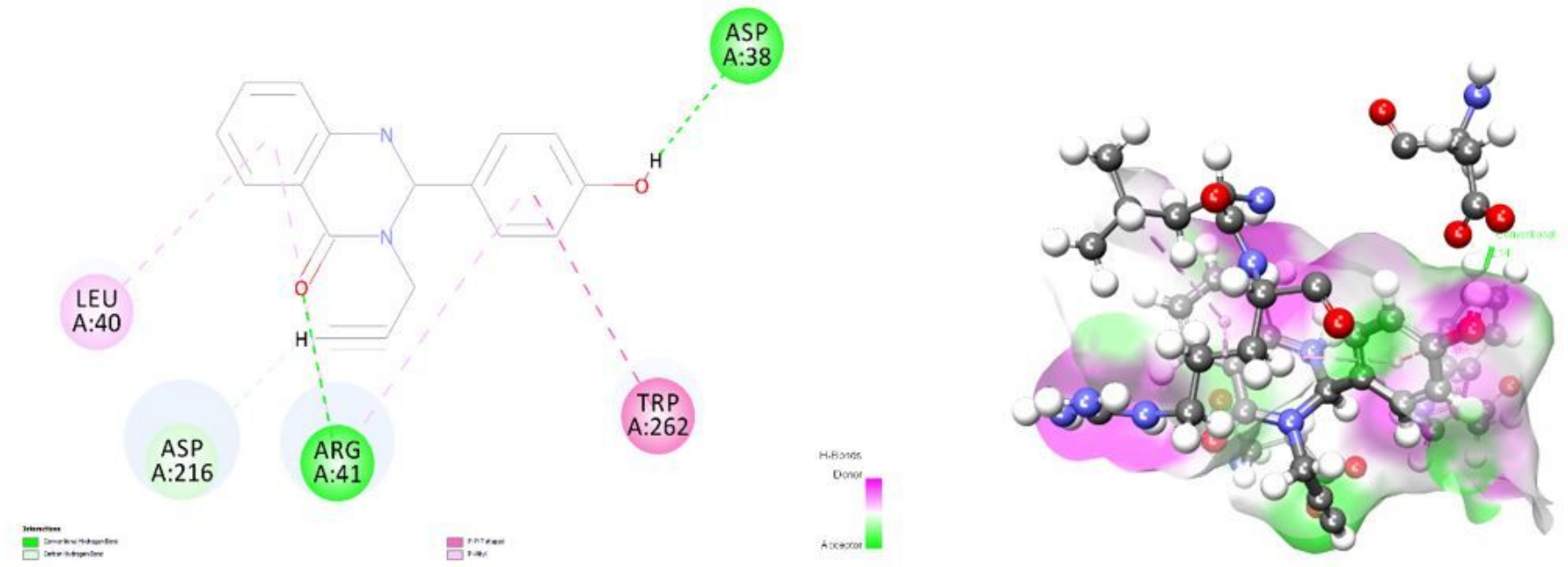

Figure 3

2D and 3D structures of $2 \mathrm{~b}$ with target 1DQY

\section{Supplementary Files}

This is a list of supplementary files associated with this preprint. Click to download.

- Capture.jpg 